\title{
Journal of Environmental
} Analysis and Progress

\section{Estimativa de radiação solar global diária com base na temperatura do ar para diferentes condições de nebulosidade em Florianópolis, Santa Catarina \\ Estimation of daily global solar radiation by air temperature under different cloudiness conditions in Florianópolis, Santa Catarina}

\author{
Flávia Roberta Auler ${ }^{\mathrm{a}}$, Rosandro Boligon Minuzzi ${ }^{\mathrm{b}}$ \\ a Agronilsen Agropecuária. Rodovia SC-435, n. 72, Terezópolis, Águas Mornas-SC, Brasil. CEP: 88150-000. E-mail: \\ fla.agro2@gmail.com. \\ b Universidade Federal de Santa Catarina-UFSC, Centro de Ciências Agrárias. Av. Admar Gonzaga, n. 1356, Itacorubi, \\ Florianópolis-SC, Brasil. CEP: 88034-001. E-mail: rbminuzzi@ hotmail.com.
}

A R T I C L E I N F O

Aceito 26 Jan 2021

Publicado 11 Fev 2022

\begin{abstract}
A B S T R A C T
Regardless of the manual or automatic form, the measurement of global solar radiation requires a high cost and frequent maintenance of the instruments, and its estimate is an alternative to obtain this type of meteorological data. The objective of this study was to evaluate four empirical models to estimate global solar radiation $(\mathrm{Rg})$ by air temperature in Florianópolis. Instrumental data of $\mathrm{Rg}$ were obtained for all days of 2016 and compared with data estimated by different methods. The analyses were made considering different cloud conditions (clear sky, partly cloudy, and cloudy) and without cloudiness distinction. The performance of each model was evaluated through the following indicators: Standard Error of Estimation, Mean Error, correlation coefficient and determination of Linear Regression, Concordance Index and Confidence Index. Rg instrumental explained about $75 \%$ of the variation of $\mathrm{Rg}$ estimated by the $\mathrm{An}$, Har and $\mathrm{Hu}$ methods and $68 \%$ by the $\mathrm{Ch}$. The performances and the correlation and determination coefficients gradually decreased with the increase in cloudiness. The $\mathrm{Hu}$ and $\mathrm{Ch}$ methods indicated a tendency for $\mathrm{Rg}$ to underestimate and overestimate the instrumental $\mathrm{Rg}$ in clear and partially cloudy sky conditions, respectively. $\mathrm{Rg}$ can be estimated using the models proposed by $\mathrm{Ch}$ and $\mathrm{Hu}$, being more efficient in the conditions of a clear and partly cloudy sky.
\end{abstract}

Keywords: Empirical model, thermal amplitude, pyranometer. 
parcialmente nublado.

Palavras-Chave: Modelo empírico, amplitude térmica, piranômetro.

Introdução

A radiação solar global $(\mathrm{Rg})$ é considerada a mais importante fonte de energia para que ocorram os processos fisiológicos e bioquímicos nas plantas. Através da fotossíntese as folhas convertem a energia radiante em energia química para que haja a produção final de matéria seca (Assis \& Mendez, 1989). No quesito das mudanças climáticas, a $\operatorname{Rg}$ para fins de geração de energia não é uma medida alternativa, mas uma necessidade urgente e global, diante de um aumento populacional iminente que demandará uma grande pressão para a produção de alimentos e energia, além de reduzir as emissões de gases de efeito estufa com a substituição das fontes de energia poluentes (Riberio et al., 2011).

Por isso, a importância de estudos que apresentem o potencial de $\mathrm{Rg}$ à superfície da Terra, uma vez que, através destas informações, pode-se executar projetos de irrigação, melhorar o aproveitamento de energia, na produção agrícola e na conservação de alimentos. Para realizar um levantamento ideal da disponibilidade de energia solar à superfície, as medições devem ser realizadas com o uso de solalímetros, piranômetros ou actinógrafos. Porém, para que estas medições sejam adequadas exige-se o uso de sistemas de aquisição de dados ou registradores e, eventualmente, de pessoas habilitadas.

Assim, independente da forma manual e, ou automática, a medida de Rg exige um custo elevado e manutenção frequente dos instrumentos, sendo a estimativa desse elemento via modelos, uma alternativa para a obtenção deste tipo de dado meteorológico, inclusive na ausência do instrumento. Os modelos empíricos são atraentes por causa de sua baixa computação, custos e dados de entrada mais acessíveis, como temperatura do ar e precipitação.

Silva, Silva \& Carvalho (2012) estimaram Rg através de modelos empíricos com uso apenas de dados de temperatura, nos quais, o modelo de Hargreaves foi eficiente para as regiões Metropolitana, do Vale do Rio Doce e na zona da Mata em Minas Gerais. No geral, concluíram que na ausência de dados instrumentais de $\mathrm{Rg}$, estimativas podem ser feitas aplicando dados da temperatura máxima e mínima do ar em modelos. Porém, para quatro localidades do Brasil (Campinas-SP, Jales-SP, Teresina-PI e PetrolinaPE), Conceição \& Marin (2007) encontraram um desempenho do modelo de Hargreaves que variou de 'regular' a 'bom'.

Os aparelhos usados nas medições de fluxos de radiação solar global ou difusa são obtidos usando um piranômetro, enquanto a medição da radiação direta é obtida com o pireliômetro, que acompanha a trajetória aparente do sol (Georgiev, Roth \& Olivares, 2004). Porém, provavelmente por seus elevados custos, esses instrumentos não fazem parte de uma estação meteorológica básica, ao contrário de instrumentos destinados à medição de temperatura, umidade relativa e precipitação.

Embora a $\mathrm{Rg}$ atinja o topo da atmosfera terrestre praticamente sem redução, existem muitas condições que alteram o fluxo que atinge a superfície da Terra, tais como cobertura de nuvens, vapor de água, aerossol e poeira ou fumaça. Os efeitos topográficos também podem ser determinantes, por exemplo, as encostas voltadas para o sul em dias de céu claro no inverno no hemisfério norte, pode receber até três vezes mais radiação solar do que encostas voltadas para o norte (Klein, 1977).

Dada a importância do monitoramento e os empecilhos para as medidas da Rg, este estudo objetivou validar modelos para a estimativa da radiação solar global na escala diária, com base na temperatura do ar.

\section{Material e Métodos}

Foram utilizados dados horários de $1^{\circ}$ de janeiro a 31 de dezembro de 2016 de radiação solar global $(\mathrm{Rg})$ e temperatura do ar obtidos das estações meteorológicas pertencentes, respectivamente, a EPAGRI (Empresa de Pesquisa Agropecuária e Extensão Rural de Santa Catarina) e ao Centro de Ciências Agrárias da UFSC, ambas localizadas no município de Florianópolis (latitude: 27,58 ${ }^{\circ}$ Sul, longitude: $48,53^{\circ}$ Oeste e altitude de 5 metros) estando distantes entre si, cerca de 200 metros. O clima do município conforme a classificação climática de Köppen é Cfa, isto é, clima subtropical, sem estação seca e temperatura média do mês mais quente acima de $22^{\circ} \mathrm{C}$ (Alvares et al., 2013).

Os dados inicialmente reportados em intervalos horários foram contabilizados para valores diários, obtendo-se valores máximos $\left(\mathrm{t}_{\max }\right)$ e mínimos $\left(\mathrm{t}_{\mathrm{mín}}\right)$ de temperatura do ar e média diária de $\mathrm{Rg}$. Os dados passaram por um processo com o propósito de eliminar possíveis inconsistências. Assim, foi usado o seguinte critério de eliminação proposto por Liu et al. (2009): dados ausentes, de $\mathrm{t}_{\max } \leq \mathrm{t}_{\min } \mathrm{e}$, ou, $\mathrm{Rg} / \mathrm{Ra}$ $>1$ ( Ra é a radiação solar no topo da atmosfera em MJ.m-2) foram eliminados, representando $41 \%$ de todos os dados analisados. A Tabela 1 apresenta algumas medidas estatísticas de posição 
e de dispersão com os dados diários válidos de $\mathrm{Rg}$ e da temperatura mínima e máxima do ar, onde percebe-se uma expressiva variação no decorrer de 2016, coerente em resposta a distinção das quatro estações do ano.

Tabela 1. Medidas de posição e de dispersão da radiação solar global $(\mathrm{Rg})$ e temperatura mínima $\left(\mathrm{t}_{\text {mín }}\right)$ e máxima diária do ar $\left(\mathrm{t}_{\max }\right)$ para Florianópolis no ano de 2016. Fonte: Auler \& Minuzzi (2020).

\begin{tabular}{lll}
$\operatorname{Rg}\left(\mathbf{M J} . m^{-2}\right)$ & $\mathbf{t}_{\min }\left({ }^{\circ} \mathbf{C}\right)$ & $\mathbf{t}_{\max }\left({ }^{\circ} \mathbf{C}\right)$ \\
\hline
\end{tabular}

\begin{tabular}{lccc}
\hline Mínimo & 2,63 & 7,7 & 14,4 \\
Máximo & 40,54 & 25,9 & 36,0 \\
Média & 21,15 & 19,3 & 25,9 \\
$1^{\circ}$ quartil & 12,11 & 16,3 & 22,6 \\
Mediana & 20,02 & 20,0 & 25,8 \\
$3^{\circ}$ quartil & 30,14 & 22,9 & 29,8 \\
\hline
\end{tabular}

Quatro equações empíricas foram utilizadas para estimar $\mathrm{Rg}$ somente com a temperatura do ar: Hargreaves (1981) (Har), Hunt et al. (1998) (Hu), Annandale et al. (2002) (An) e Chen et al. (2004) (Ch) (Tabela 2).

Tabela 2. Modelos empíricos utilizados neste estudo para a estimativa da radiação solar global. Fonte: Auler \& Minuzzi (2020).

\begin{tabular}{llll}
\hline Modelo & Método & Equação* & Coeficientes \\
\hline Annandale et al. (2002) & $\mathrm{An}$ & $\mathrm{Rg}=\mathrm{a} \cdot\left(1+2,7 \times 10^{-5} \cdot \mathrm{Alt}\right) \cdot\left(\Delta \mathrm{T}_{1}\right)^{0,5} \cdot \mathrm{Ra}$ & $\mathrm{a}=0,159$ \\
Hargreaves (1981) & $\mathrm{Har}$ & $\mathrm{Rg}=\mathrm{a} \cdot\left(\Delta \mathrm{T}_{1}\right)^{0,5} \cdot \mathrm{Ra}$ & $\mathrm{a}=0,161$ \\
Chen et al. (2004) & $\mathrm{Ch}$ & $\mathrm{Rg}=\left(\mathrm{a} \cdot\left(\Delta \mathrm{T}_{1}\right)^{0,5}+\mathrm{b}\right) \cdot \mathrm{Ra}$ & $\mathrm{a}=0,384 ; \mathrm{b}=-0,369$ \\
Hunt et al. (1998) & $\mathrm{Hu}$ & $\mathrm{Rg}=\mathrm{a} \cdot\left(\Delta \mathrm{T}_{1}\right)^{0,5} \cdot \mathrm{Ra}+\mathrm{b}$ & $\mathrm{a}=0,275 ; \mathrm{b}=-0,363$ \\
\hline
\end{tabular}

$* \mathrm{Rg}=$ radiação solar incidente na superfície $\left(\mathrm{MJ} \mathrm{m}^{-2} \mathrm{dia}^{-1}\right) ; \mathrm{Ra}=$ radiação solar incidente extraterrestre (MJ $\left.\mathrm{m}^{-2} \mathrm{dia}^{-1}\right) ;$ Alt. $=$ altitude local $(\mathrm{m}) ; \Delta \mathrm{T}_{1}=$ diferença entre a temperatura máxima e mínima do dia $\left({ }^{\circ} \mathrm{C}\right) ; \mathrm{a}, \mathrm{b}=$ coeficientes de calibração dos modelos empíricos (adimensionais), conforme obtidos em estudo de Silva et al. (2012).

O modelo de Hargreaves (1981) é amplamente utilizado em razão da sua simplicidade, inclusive recomendado no boletim da FAO-56 para ser usado quando há ausência de dados de $\mathrm{Rg}$ ou são de qualidade duvidosa (Allen et al., 1998). A partir do modelo inicial de Hargreaves (1981), novas modificações foram sendo propostas, como Chen et al. (2004) propuseram relacionar $\mathrm{Rg}$ de maneira logarítmica com a raiz quadrada da diferença $\left(\Delta \mathrm{T}_{1}\right)$; Annandale et al. (2002) introduziram uma nova variável para a correção da altitude.

A avaliação da estimativa de $\mathrm{Rg}$ pelos referidos métodos foi realizada por meio do erro padrão da estimativa (EPE), do erro médio (EM), dos coeficientes de correlação $(r)$ e de determinação $\left(\mathrm{R}^{2}\right)$ da regressão linear, do índice de concordância (d) e do índice de confiança (c).

O Índice de Concordância (d) (Willmott, 1981), descrito na Equação 1, varia de 0 a 1 e representa o quanto os valores de $\mathrm{Rg}$ obtidos pela forma padrão (dado instrumental) se ajustam aos valores estimados pelos modelos, sendo que, valores próximos de 1 , indicam uma concordância perfeita.

$d=1-\left[\frac{\sum_{i=1}^{N}\left(Y_{i}-X_{i}\right)^{2}}{\sum_{i=1}^{N}\left(\left|Y_{i}-\bar{X}\right|+\left|X_{i}-\bar{X}\right|\right)^{2}}\right]$

onde $\mathrm{X}_{\mathrm{i}}=$ são os valores de $\mathrm{Rg}$ medido por instrumento; $\bar{X}=$ é a média dos valores de $\mathrm{Rg}$ medido por instrumento; $Y_{i}=$ são os valores de $\mathrm{Rg}$ estimados pelos modelos; e $\mathrm{N}=$ é o número de dados de Rg.

Correlativamente, para a análise da confiabilidade de $\mathrm{Rg}$ estimada pelos modelos, considerou-se o Índice de Confiança (c), proposto por Camargo \& Sentelhas (1997), conforme a Equação 2.

$c=r . d$

O critério adotado para interpretar os valores de c consta na Tabela 3.

Tabela 3. Índices de confiança e desempenho da $\mathrm{Rg}$ estimada pelos modelos. Fonte: Camargo \& Sentelhas (1997).

\begin{tabular}{cc}
\hline $\begin{array}{c}\text { Índice de confiança } \\
\text { (c) }\end{array}$ & Desempenho \\
\hline$>0,86$ & Ótimo \\
0,76 a 0,85 & Muito Bom \\
0,66 a 0,75 & Bom \\
0,61 a 0,65 & Mediano \\
0,51 a 0,60 & Sofrível \\
0,41 a 0,50 & Mau \\
$\leq 0,40$ & Péssimo \\
\hline
\end{tabular}

O cálculo para o erro padrão da estimativa (EPE) foi obtido utilizando a Equação 3.

$E P E=\sqrt{\left(\frac{\sum_{i=1}^{N}\left(Y_{i}-X_{i}\right)^{2}}{N-1}\right)}$ 
O Erro Médio (EM), representa a diferença média de $\mathrm{Rg}$ estimada pelos modelos com os valores registrados por instrumento. Assim, o EM indica a possível tendência de Rg estimada pelos modelos em superestimar $(E M>0)$ ou subestimar $(E M<0)$ a $\mathrm{Rg}$ instrumental, sendo calculado conforme Equação 4.

$$
E M=\sum_{i=1}^{N}\left(Y_{i}-X_{i}\right)
$$

A avaliação das estimativas de $\operatorname{Rg}$ foram feitas considerando: a) a nebulosidade de cada dia, definido pelo Índice de Claridade $\left(\mathrm{I}_{\mathrm{k}}\right)$, que relaciona $\mathrm{Rg}$ com $\mathrm{Ra}: 0<\mathrm{I}_{\mathrm{k}}<0,3$ (Céu nublado); $0,3 \leq \mathrm{I}_{\mathrm{k}} \leq 0,65$ (Céu parcialmente nublado) e $0,65<\mathrm{I}_{\mathrm{k}}<1,0$ (Céu claro) e, b) todos os dias do período em estudo (sem distinção das condições de nebulosidade).

\section{Resultados e Discussão}

A avaliação da estimativa de Rg através dos modelos empíricos com dados de temperatura do ar são apresentadas na Tabela 4. Os valores do índice de confiança (c) indicam que na análise geral dos dados os modelos propostos por Hunt et al. (1998) e Chen et al. (2004) mostraram um desempenho 'muito bom' para a estimativa de Rg. Quando analisados os coeficientes de determinação $\left(\mathrm{R}^{2}\right)$, os valores apontam que o $\mathrm{Rg}$ por instrumento explicou de $71 \%$ a $74 \%$ da variação de $\mathrm{Rg}$ estimada pelos métodos, inclusive o de Annandale et al. (2002) e o de Hargreaves (1981), que mostraram um desempenho 'sofrível'. Em relação ao EPE, as estimativas dos modelos com melhor desempenho apresentaram uma variação de 5 a 5,8 MJ.m ${ }^{-2}$ em relação ao $R g$ do instrumento.

Tabela 4. Métodos utilizados para a avaliação da estimativa da Rg diária segundo Annandale et al. (2002) (An), Hargreaves (1981) (Har), Chen et al. (2004) (Ch) e Hunt et al. (1998) (Hu) para todas as datas válidas de 2016 e de acordo com a nebulosidade para Florianópolis. Fonte: Auler \& Minuzzi (2020).

\begin{tabular}{|c|c|c|c|c|c|c|c|}
\hline \multirow{2}{*}{ Métodos } & EPE & EM & d & $\mathbf{r}$ & $\mathbf{R}^{2}$ & c & Desempenho \\
\hline & \multicolumn{7}{|c|}{ Geral (sem distinção de nebulosidade) } \\
\hline $\mathrm{An}$ & 9,78 & $-7,36$ & 0,68 & 0,85 & 0,71 & 0,58 & Sofrível \\
\hline $\mathrm{Hu}$ & 5,78 & 2,33 & 0,89 & 0,85 & 0,71 & 0,76 & Muito Bom \\
\hline Har & 9,63 & $-7,19$ & 0,69 & 0,85 & 0,71 & 0,58 & Sofrível \\
\hline $\mathrm{Ch}$ & 4,99 & \multicolumn{5}{|c|}{ Céu claro } & Muito Bom \\
\hline An & 13,44 & $-12,76$ & 0,56 & 0,87 & 0,75 & 0,49 & Sofrível \\
\hline $\mathrm{Hu}$ & 4,11 & $-1,42$ & 0,92 & 0,87 & 0,75 & 0,80 & Muito Bom \\
\hline Har & 13,24 & $-12,57$ & 0,56 & 0,87 & 0,75 & 0,49 & Sofrível \\
\hline \multirow[t]{2}{*}{$\mathrm{Ch}$} & 5,36 & $-2,75$ & 0,88 & 0,83 & 0,68 & 0,73 & Bom \\
\hline & \multicolumn{7}{|c|}{ Céu parcialmente nublado } \\
\hline $\mathrm{An}$ & 5,50 & $-4,37$ & 0,76 & 0,84 & 0,71 & 0,64 & Mediano \\
\hline $\mathrm{Hu}$ & 5,96 & 4,56 & 0,81 & 0,84 & 0,71 & 0,68 & Bom \\
\hline Har & 5,37 & $-4,21$ & 0,77 & 0,84 & 0,71 & 0,65 & Mediano \\
\hline \multirow[t]{2}{*}{$\mathrm{Ch}$} & 4,71 & 1,03 & 0,87 & 0,78 & 0,61 & 0,68 & Bom \\
\hline & \multicolumn{7}{|c|}{ Céu nublado } \\
\hline An & 2,88 & 1,81 & 0,63 & 0,65 & 0,42 & 0,41 & Sofrível \\
\hline $\mathrm{Hu}$ & 9,38 & 8,42 & 0,06 & 0,65 & 0,42 & 0,04 & Péssimo \\
\hline Har & 2,98 & 1,93 & 0,62 & 0,65 & 0,42 & 0,40 & Péssimo \\
\hline $\mathrm{Ch}$ & 5,49 & 2,90 & 0,47 & 0,38 & 0,15 & 0,18 & Péssimo \\
\hline
\end{tabular}

$\mathrm{EPE}=$ erro padrão de estimativa; $\mathrm{EM}=$ erro médio; $d=$ =índice de concordância; $r=$ coeficiente de correlação; $\mathrm{R}^{2}=$ coeficiente de determinação; $\mathrm{c}=$ índice de confiança.

Fatores astronômicos e geográficos à parte, a $\mathrm{Rg}$ é fortemente modificada pela cobertura de nuvens, albedo da superfície e absorção e turbidez atmosférica. Por isso, nas análises de acordo com a cobertura de nuvens os resultados diferem entre si, mas mantendo a mesma sequencia de desempenho dos modelos ao observado na análise sem distinção de nebulosidade, isto é, o de Hunt et al. (1998) e o de Chen et al. (2004), com os melhores desempenhos. A começar pelas condições de céu claro, as estimativas oriundas do modelo de Hunt et al. (1998) mantiveram o desempenho 'muito bom', mas o de Chen et al. (2004) diminuiu uma classe, tendo um 'bom' desempenho. Os valores de $\mathrm{R}^{2}$ mostram que o Rg por instrumento explicou cerca $75 \%$ da variação de $\mathrm{Rg}$ estimada pelos métodos de An, Har e Hu e $68 \%$ pelo de Chen et al. (2004), mesmo tendo um desempenho melhor que o de Hargreaves (1981) e Annandale et al. (2002), mostrando a importância em utilizar 
diferentes métodos estatísticos em análises comparativas de dados.

Os desempenhos e os coeficientes de correlação e de determinação diminuíram gradativamente com o aumento da nebulosidade. Mesma tendência observada para o EPE, onde

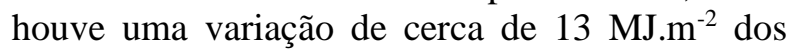
valores estimados obtidos para céu claro até em torno de $3 \mathrm{MJ} \cdot \mathrm{m}^{-2}$ nas condições de céu nublado. Esta tendência do EPE é coerente nas suas devidas proporções, pois em condições de céu claro têmse maiores valores de $\mathrm{Rg}$ que diminuem a medida que aumenta a nebulosidade.

Em condições de céu nublado os desempenhos de todos os modelos foram de 'sofrível' a 'péssimo' com o Rg instrumental não explicando nem a metade da variação do $\mathrm{Rg}$ estimado $\left(\mathrm{R}^{2}\right.$ de 0,15 a 0,42$)$. Iziomon \& Mayer (2001) encontraram esta mesma tendência na precisão das estimativas de $\mathrm{Rg}$, mesmo que elas tenham sido na escala mensal e com modelo baseado na cobertura de nuvens.

Em condições de nebulosidade para a (2017) encontraram melhores estimativas nos modelos de estimativa de $\mathrm{Rg}$ que necessitam de dados de insolação (horas de brilho solar) do que nos de cobertura de nuvens. Mesmo assim, com o aumento da nebulosidade ambos os modelos tiveram decréscimo gradativo na precisão, apesar de que em condições de céu parcialmente nublado os resultados ainda foram satisfatórios. Como estes resultados foram semelhantes aos obtidos para Florianópolis, mesmo com modelos diferentes, uma das hipóteses apontadas por Didari \& Zand-Parsa (2017) para essa tendência no desempenho de acordo com a nebulosidade, também pode ser aplicada para modelos que necessitam apenas de temperatura do ar, isto é, a estimativa de $\mathrm{Rg}$ em dias de nebulosidade não depende apenas da cobertura de nuvens, mas também de outros parâmetros relacionados as propriedades das nuvens.

Os modelos de estimativa de $\mathrm{Rg}$ baseados na temperatura consideram as hipóteses de que condições de céu limpo aumentam a temperatura máxima devido a maior emissão de radiação de ondas curtas embora resultem num decréscimo da temperatura mínima devido a maior perda de radiação de ondas longas para a atmosfera, resultando em maior amplitude térmica diária. Nas condições de maior nebulosidade, acarretará em menor temperatura máxima e maior temperatura mínima devido ao aumento da emissão de ondas longas pelas nuvens, ocasionando menor amplitude térmica. região central e sul do Irã, Didari \& Zand-Parsa

Besharat, Dehghan \& Faghih (2013) destacam que a amplitude térmica do $\operatorname{ar}(\Delta \mathrm{T})$ pode afetar a validação dos modelos, onde maiores valores de $\Delta \mathrm{T}$ geralmente resulta numa melhor precisão. Isto significa que os modelos baseados na temperatura do ar são mais aplicáveis em áreas com maior amplitude térmica. Seguindo este raciocínio, os melhores desempenhos de $\mathrm{Rg}$ obtidos em Florianópolis para céu claro são coerentes, tendo em vista que, são nestas condições que normalmente se tem maiores amplitudes térmicas.

Os valores do erro médio (EM) para os modelos com melhores desempenhos ( $c \geq 0,66$ ), sendo o de Hunt et al. (1998) e de Chen et al. (2004), indicaram uma tendência do $\mathrm{Rg}$ em subestimar (-1,42 MJ.m e $^{-2} \quad-2,75$ MJ.m $\left.{ }^{-2}\right)$ e superestimar $\left(4,56 \mathrm{MJ} . \mathrm{m}^{-2}\right.$ e $\left.1,03 \mathrm{MJ} \cdot \mathrm{m}^{-2}\right)$ o $\mathrm{Rg}$ instrumental nas condições de céu claro e parcialmente nublado, respectivamente (Tabela 3 ).

Porém, na análise sem distinção de nebulosidade houve uma diferença na tendência de estimativa entre estes dois modelos. Os gráficos de dispersão dos pontos entre $\mathrm{Rg}$ instrumental com $\mathrm{Rg}$ estimada para qualquer condição de nebulosidade pelos modelos de Hunt et al. (1998) e de Chen et al. (2004), em torno da reta $1: 1$, detalham esta análise de tendência na Figuras 1a e 1b, respectivamente. Nesta análise geral, a Figura 1a mostra que para valores de Rg estimados abaixo de $30 \mathrm{MJ} . \mathrm{m}^{-2}$ e de $20 \mathrm{MJ} . \mathrm{m}^{-2}$ aproximadamente, pelo modelo de Hunt et al. (1998) e de Chen et al. (2004), tendem a ser superestimados, ocorrendo tendência contrária para estimativas de valores acima dos referidos limiares.

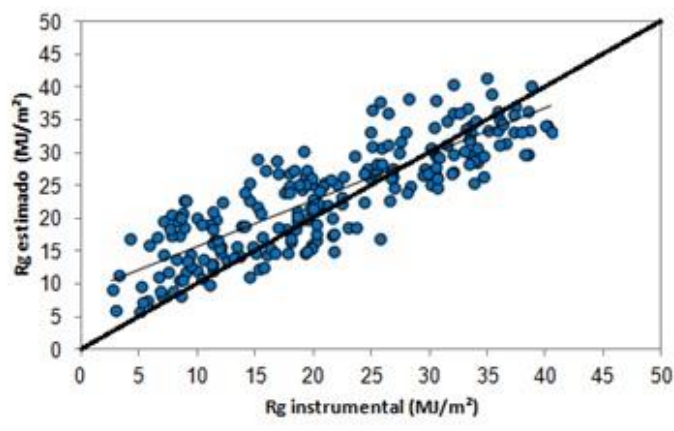




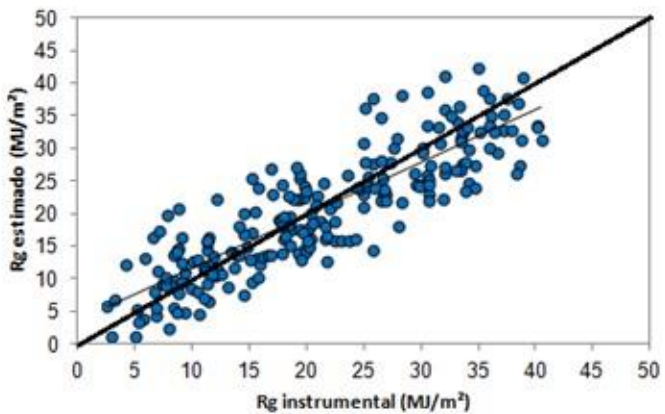

b

Figura 1. Relação entre Rg padrão (instrumental) e o estimado pelos modelos propostos em a. Hunt et al. (1998); Chen et al. (2004), na análise sem distinção de nebulosidade em b. Fonte: Auler \& Minuzzi (2020).

Souza et al. (2017) utilizaram 15 modelos para a estimativa de $\mathrm{Rg}$ necessitando apenas de temperatura do ar para o estado do Mato Grosso e, mesmo calibrando os coeficientes de alguns dos modelos que foram semelhantes aos utilizados neste estudo, a precisão de alguns dos 29 locais analisados foi abaixo aos dados obtidos em Florianópolis. Soma-se a isto, que de todos os dias válidos para a análise, em 13,5\% o céu esteve nublado, 40,5\% parcialmente nublado e $46 \%$ de céu claro em Florianópolis, isto é, cerca de 86,5\% dos dias analisados, a estimativa de $\mathrm{Rg}$ teve um desempenho de "bom" a "muito bom" usufruindo dos modelos propostos por Hunt et al. (1998) e Chen et al. (2004) que tiveram os melhores desempenhos.

Borges et al. (2010) apresentaram o modelo de Hargreaves (1981) com bons resultados para a região litorânea da Bahia, contrariando ao desempenho observado neste estudo para Florianópolis e mostrando a importância em validar modelos para diferentes locais, inclusive aqueles que necessitam de mais dados para sua estimativa.

Massignam (2007) ao validar três modelos dependentes da temperatura do ar (Richardson, Hargreaves e Bristow-Campbell) para 14 locais de Santa Catarina, encontrou uma variação no desempenho, que foi de 'mau' a 'sofrível' para Florianópolis. Com Hargreaves, o pesquisador encontrou que a $\mathrm{Rg}$ medida explicou 49\% da variação de $\mathrm{Rg}$ estimada pelo referido método. Desempenho insatisfatório do modelo de Hargreaves também foi encontrado por Fernandes et al. (2018) para o estado de Goiás. Para o município de Barbalha, no Ceará, mesmo estimando o coeficiente de ajuste exigido no método de Hargreaves e Samani, em função da altitude do local, Lêdo et al. (2012) encontraram um 'péssimo' desempenho na estimativa de Rg, ou seja, assim como no estudo de Souza et al.
(2017), o ajuste dos coeficientes não significa, necessariamente, uma melhora no desempenho em modelos de estimativa de Rg.

Liu \& Scott (2001) avaliaram a desempenho de nove modelos para estimativa da $\mathrm{Rg}$ diária baseados em dados de chuva e/ou temperatura do ar para 39 locais da Austrália. No geral, modelos que necessitam de dados de temperatura juntamente com chuva foram os de melhor desempenho independente se os coeficientes dos modelos foram desenvolvidos para cada local ou usados de outras regiões independente da sua localização (litorânea ou interiorana).

\section{Conclusão}

A radiação solar global diária pode ser estimada em Florianópolis, usando dados de temperatura do ar nos modelos propostos por Hunt et al. (1998) e Chen et al. (2004), sendo mais eficientes nas condições de céu claro e parcialmente nublado.

\section{Agradecimentos}

Os autores agradecem à EPAGRI (Empresa de Pesquisa Agropecuária e Extensão Rural de Santa Catarina) e ao Centro de Ciências Agrárias da UFSC pela disponibilidade dos dados meteorológicos.

\section{Referências}

Allen, R. G.; Pereira, L. S.; Raes, D.; Smith, M. 1998. Crop evapotranspiration: guidelines for computing crop water requirements. Irrigation and Drainage, Paper 56. Rome: FAO, 300p.

Alvares, C. A.; Stape, J. L.; Sentelhas, P. C.; Moraes, G.; Leonardo, J.; Sparovek, G. 2013. Köppen's climate classification map for Brazil. Meteorol. Z., 22, 6, 711-728.

Annandale, J. G.; Jovanovic, N.; Benade, N. Allen, R. G. 2002. Software for missing data error analysis of Penman-Monteith reference evapotranspiration. Irrig. Sci., 21, 57- 67.

Assis, F. N.; Mendez, M. E. G. 1989. Relação entre radiação fotossinteticamente ativa e radiação global. Pesq. Agropec. Bras., 7, 24, 797-800.

Besharat, F.; Dehghan, A. A.; Faghih, A. R. 2013. Empirical models for estimating global solar radiation: A review and case study. Renew. Sust. Energ. Rev., 21, 798-821.

Borges, V. P.; Oliveira, A. S.; Coelho Filho, M. A.; Silva, T. S. M. da; Pamponet, B. M. 2010. Avaliação de modelos de estimativa da radiação solar incidente em Cruz das Almas, 
Bahia. Rev. Bras. Eng. Agríc. Ambient., 14, 1, 74-80.

Camargo, A. P.; Sentelhas, P. C. 1997. Avaliação do desempenho de diferentes métodos de estimativa da evapotranspiração potencial no Estado de São Paulo. Rev. Bras. Agromet., 5, 89-97.

Chen, R. S.; Ersi, K; Yang, J. P.; Lu, S. H.; Zhao, W. Z. 2004. Validation of five global radiation models with measured daily data in China. Energy Convers. Manag., 45, 17591769.

Conceição, M. A. F.; Marin, F. R. 2007. Avaliação de modelos para a estimativa de valore diários da radiação solar global com base na temperatura do ar. Rev. Bras. Agromet., 15, 103-108.

Didari, D.; Zand-Parsa, S. 2017. Estimation of daily global solar irradiation under different sky conditions in central and southern Iran. Theor. Appl. Climatol., 127, 587-596.

Fernandes, D. S.; Heinemann, A. B.; Amorim, A. O.; Paz, R. L. F. da. 2018. Estimativa da radiação solar global com base em observações de temperatura para o estado de Goiás. Rev. Bras. de Meteorol., 33, 558-566.

Georgiev, A.; Roth, P.; Olivares, A. 2004. Sun following system adjustment at the UTFSM. Energy Convers. Manage., 45, 1795-1806.

Hargreaves, G. H. 1981. Responding to tropical climates. The 1980-81 Food and climate review, the food and climate forum. Boulder: Aspen Institute for Humanistic Studies, pp. 29-32.

Hunt, L. A.; Kuchar, L.; Swanton, C. J. 1998. Estimation of solar radiation for use in crop modeling. Agric. For. Meteorol., 91, 293300.

Iziomon, M. G.; Mayer, H. 2001. Performance of solar radiation models - a case study. Agric. For. Meteorol., 110, 1-11.
Klein, S .A. 1977. Calculation of monthly average insolation on tilted surfaces. Sol. Energy, 19, 325-329.

Lêdo, E. R. F.; Silva, M. G. da; Nogueira, D. H.; Arraes, F. D. D. 2012. Avaliação de modelo de estimativa da radiação solar global com base na amplitude térmica. Conex. Ci. E Tecnol., 6, 15-26.

Liu, X.; Mei, X.; Li, Y.; Wang, Q.; Jensen, R. J.; Zhang, Y.; Porter, J. R. 2009. Evaluation of temperature-based global solar radiation models in China. Agric. For. Meteorol., 149, 1433-1446.

Liu, D. L.; Scott, B. J. 2001. Estimation of solar radiation in Australia from rainfall and temperature observations. Agric. For. Meteorol., 106, 41-59.

Massignam, A. M. 2007. Estimativa da radiação solar em função da amplitude térmica. Anais XV Congresso Brasileiro de Agrometeorologia, Aracajú, SE, Brasil, SBAGRO.

Riberio, R. M.; Dias, L. A. S.; Berger, P.G.; Dias, D. C. F. S. 2011. Agroenergia na mitigação das mudanças climáticas globais, na segurança energética e na promoção social. Viçosa, MG: Suprema, 201p.

Silva, C. R.; Silva, V. J.; Carvalho, H. P. 2012. Radiação solar estimada com base na temperatura do ar para três regiões de Minas Gerais. Rev. Bras. Eng. Agríc. Ambient., 16, 3, 281-288.

Souza, A. P. de; Silva, A. C. da; Tanaka, A. A.; Uliana, E. M.; Almeida, F. T.; Klar, A. E.; Gomes, W. A. 2017. Global radiation by simplified models for the state of Mato Grosso, Brazil. Pesq. Agropec. Bras., 52, 4, 215-227.

Willmott, C. J. 1981. On the validation of models. Phys. Geogr., 2, 2, 184-194. 